\title{
Maximum Variance Combined with Adaptive Genetic Algorithm for Infrared Image Segmentation
}

\author{
Huixuan $\mathrm{Fu}$ \\ College of Automation \\ Harbin Engineering University, \\ Harbin, China \\ fuhuixuan@hrbeu.edu.cn \\ Liangliang Han \\ College of Automation \\ Harbin Engineering University, \\ Harbin, China \\ hanliangliang@hrbeu.edu.cn
}

\author{
Yuchao Wang* \\ College of Automation \\ Harbin Engineering University, \\ Harbin, China \\ wangyuchao@hrbeu.edu.cn \\ * Corresponding Author
}

\begin{abstract}
Maximum Variance Image Segmentation method (Otsu) is a popular non-parametric method in image segmentation. However, it is large amount of computation and poor real-time quality have limited its further application. To solve these problems, a new approach based on an adaptive genetic algorithm (AGA) and Otsu are proposed, which using between-class variance as fitness function, automatically adjusts the optimal threshold. The adaptive genetic algorithm selects crossover probability and mutation probability according to the fitness values, reduces the convergence time and improves the precision of genetic algorithm, insuring the accuracy of parameter selection. The experimental results show that the proposed method is better than the original Otsu, the AGA-Otsu can provide better effectiveness on experiments of infrared image segmentation, decrease processing time.
\end{abstract}

Keywords- Image segmentation; Otsu; Adaptive Genetic Algorithm; Infrared image; Optimal threshold

\section{INTRODUCTION}

Image segmentation is considered as an important basic operation for meaningful analysis and interpretation of image acquired. Image segmentation is a process of dividing an image into a number or sub-areas with different characteristics and extracting the interested objects. One of the methods of image segmentation is the threshold-based method which divides the image into several areas by one or more thresholds and considers the pixels belonging to the same area as a separate object. Threshold-based method is definitely one of the most popular segmentation approaches to extract objects from images, and more particularly in infrared images [1]. The advantages of the threshold-based method are its simplicity and easy implementation while the difficulties lie in how to select the best threshold to ensure a satisfactory segmentation result. Many threshold-based methods have been proposed by a lot of scholars. Pun [2] proposed an algorithm based on entropy. Maximum classbetween variance algorithm (also known as the Otsu algorithm) [3], introduced a nonparametric and unsupervised method for image segmentation. The minimum error algorithm [4,5], Adaptive minimum error algorithm [6], Fuzzy Cluster [7-9] etc. Maximum classbetween variance algorithm uses image histogram to determine the best segmentation threshold between the object and background of an image. It can well pledges the result, which doesn't need priori knowledge [10]. However, the solution of optimization threshold is gotten by exhaustive search and the process costs too much time.

Genetic algorithms (GA) are robust search and optimization techniques which are finding application in a number of practical problems [11, 12]. The basic operating principles of GA are based on the principles of natural evolution. The robustness of GA requires little knowledge of the problem itself and does not require that the search space is differentiable or continuous. GA for reproduction can provide the solution for optimizing parameters. Adaptive genetic algorithm (AGA) is a type of GA of which probabilities of crossover and mutation are adaptively adjusted according to the fitness values of individuals [12].

In order to avoid the shortcomings of the Otsu algorithm time-consuming, a new approach based on an adaptive genetic algorithm (AGA) and Otsu is proposed, which using between-class variance as fitness function, automatically adjusts the optimum threshold, this method selects crossover probability and mutation probability according to the fitness values, insuring the accuracy of parameter selection, decrease the processing time. 


\section{OTSU ALGORITHM}

The Otsu algorithm is proposed by Nobuyuki Otsu [3], based on the maximum variance between classes. According to the histogram of an image, Otsu method chooses the maximum between class are variance from the background as the criterion of threshold. Due its good segmentation effect, it has become one of the most widely used threshold-based segmentation methods. The principle of the algorithm can be described as follows.

Let the pixels of a given image be represented in $\mathrm{L}$ gray levels. The collection of all gray values of the image is $G=\{0,1,2, \ldots, L-1\}$. The number of pixels at level $i$ is denoted by $n_{i}$, the total number of pixels is:

$$
N=\sum_{i=0}^{L-1} n_{i}
$$

In order to simplify the discussion, the gray-level histogram is normalized and regarded as a probability distribution:

$$
P_{i}=n_{i} / N
$$

where $P_{i} \geq 0, \sum_{i=0}^{L-1} P_{i}=1$

Supposing the image is segmented into two regions by the threshold which has the gray value $t$. The region contains pixels have the gray value between 0 to $t-1$ is defined as $C_{0}$, which represents the background of the image. The region contains pixels and have the gray value between $t$ to $L-1$ which is defined as $C_{1}$, which represents the object in the image.

The probability of $C_{0}$ and $C_{1}$ are:

$$
\begin{gathered}
\omega_{0}=\sum_{i=0}^{t-1} P_{i}=\omega(t) \\
\omega_{1}=\sum_{i=t}^{L-1} P_{i}=1-\omega(t)
\end{gathered}
$$

The mean of $C_{0}$ and $C_{1}$ gray values are:

$$
\begin{array}{r}
\mu_{0}=\frac{\sum_{i=0}^{t-1} i P_{i}}{\omega_{0}} \\
\mu_{1}=\frac{\sum_{i=t}^{L-1} i P_{i}}{\omega_{1}}
\end{array}
$$

The average gray value of whole image is:

$$
\mu=\sum_{i=0}^{L-1} i P_{i}
$$

$C_{0}$ and $C_{1}$ class variance by the following formula:

$$
\begin{gathered}
\sigma_{0}^{2}=\sum_{i=0}^{t-1} \frac{\left(i-\mu_{0}\right)^{2} \cdot P_{i}}{\omega_{0}} \\
\sigma_{1}^{2}=\sum_{i=t}^{m-1} \frac{\left(i-\mu_{1}\right)^{2} \cdot P_{i}}{\omega_{1}}
\end{gathered}
$$

Define class variance $\sigma_{\omega}^{2}$, between-class variance $\sigma_{B}^{2}$ and $\sigma_{T}^{2}$ population variance as:

$$
\begin{gathered}
\sigma_{\omega}^{2}=\omega_{0} \sigma_{0}^{2}+\omega_{1} \sigma_{1}^{2} \\
\sigma_{B}^{2}=\omega_{0}\left(\mu_{0}-\mu\right)^{2}+\omega_{1}\left(\mu_{1}-\mu\right)^{2}=\omega_{0} \omega_{1}\left(\mu_{1}-\mu_{0}\right)^{2}
\end{gathered}
$$

$$
\sigma_{T}^{2}=\sigma_{\omega}^{2}+\sigma_{B}^{2}
$$

In the expression of $\sigma_{\omega}^{2}$ and $\sigma_{B}^{2}$ are contained threshold $\mathrm{t}$, it is possible to introduce a soft decision rule:

$$
\eta(t)=\frac{\sigma_{B}^{2}}{\sigma_{T}^{2}}
$$

Then the problem is reduced to an optimization problem to search for a threshold $t$ that maximizes one of the object functions (the criterion measures). This standpoint is motivated by a conjecture that threshold classes will be separated in gray levels, and conversely, a threshold giving the best separation of classes in gray levels will be the best threshold.

It is noticed that $\sigma_{\omega}^{2}$ and $\sigma_{B}^{2}$ are functions of threshold level $t$, but $\sigma_{T}^{2}$ is independent of $t$. Therefore, $\eta(t)$ is the simplest measure with respect to $t$. Thus, adopt $\eta(t)$ as the criterion measure to evaluate the threshold at level $t$ The value in the range of pixels gray levels in image is $t$, when the results of $\eta(t)$ reaches a maximum, then take $t^{*}$ as the best threshold:

$$
\max _{0 \leq t \leq m-1} \eta(t)=\max \eta\left(t^{*}\right)
$$

\section{OTSU COMBINED WITH ADAPTIVE GENETIC ALGORITHM}

Otsu solving process finds an optimal solution in the solution space, making the maximum between-class variance. In order to find the variable $t$ which causes $\eta(t)$ to maximum value, the algorithm calculates a variance for each gray value in the gray level collection $G$ and thus it is hardly for Otsu to achieve real-time processing requirements.

Simple Genetic Algorithms (SGA) is firstly proposed by John H. from the Michigan University in America. As a newly developed optimization algorithm, the genetic algorithm is derived from the theory of Darwinism and genetics. Simple Genetic Algorithm is characterized by its current effectiveness, strong robustness, and simple implementation. But Simple genetic algorithm has premature convergence problem[13,14]. Therefore, the goals with adaptive probabilities of crossover and mutation are to maintain the genetic diversity in the population and prevent the genetic algorithms to converge prematurely to local minima. Adaptive genetic algorithm can get better solution for the problem by adjusting crossover probability $P_{c}$ and the mutation probability $P_{m}$ dynamically according to the individuals fitness[15]. Adaptive genetic algorithm is used to search for the optimal Otsu parameter, improve search speed and reduce calculation.

\section{A. Algorithm Design}

\section{1) Code and Fitness Function}

Use binary encode. For the gray-level from 0 to 255 , individual denoted by chromosome is coded into 8 bit as a threshold. Fitness function is a key factor to obtain threshold through GA. Define between-class variance $\sigma_{B}^{2}$ as the fitness function. Use Formula (11) to calculate the individual fitness value. 


\section{2) Selection Operation}

The stochastic tournament selection is implemented for the current population for reproduction.

\section{3 ) Crossover and Mutation Operation}

Crossover and Mutation Operation, create new offspring by performing crossover and mutation operations. The probabilities of crossover and mutation can be defined in the following forms [15]:

$$
\begin{gathered}
P_{c}=\left\{\begin{array}{cc}
P_{c_{1}}-\frac{\left(P_{c_{1}}-P_{c_{2}}\right)\left(f i t_{i}-f i t_{\text {avg }}\right)}{\left(f i t_{\max }-f i t_{\text {avg }}\right)}, & f i t_{i} \geq f i t_{\text {avg }} \\
P_{c_{1}}, & f i t_{i}<f i t_{\text {avg }}
\end{array}\right. \\
P_{m}=\left\{\begin{array}{cc}
P_{m_{1}}-\frac{\left(P_{m_{1}}-P_{m_{2}}\right)\left(f i t_{\max }-f i t_{i}\right)}{\left(f i t_{\max }-f i t_{\text {avg }}\right)}, & f i t_{i} \geq f i t_{\text {avg }} \\
P_{m_{1}}, & f i t_{i}<f i t_{\text {avg }}
\end{array}\right.
\end{gathered}
$$

where $P_{c_{1}}$ is the maximum probability of crossover, $P_{c 2}$ is the minimum probability of crossover, fit $_{i}$ is the larger fitness value of the two individuals selected for crossover, $P_{m_{1}}$ is the maximum probability of mutation, $P_{m 2}$ is the minimum probability of mutation, fit $_{\text {max }}$ is the maximum fitness value of the current population, fit $_{\text {avg }}$ is the average fitness value of the current population, and fit is the fitness value of the individual to mutate.

\section{B. Algorithm Realization}

The Algorithm of infrared image segmentation based on Otsu and adaptive genetic algorithm consists of the following steps:

Step1: Initialization population, define the adaptive genetic algorithm's operational parameters (the number of variables, search domain of each variable, the number of individuals in population, maximum number of evolution generations, maximum and minimum mutation probability, and maximum and minimum crossover probability), iterative times $k=1$, generate an random initial population.

Step2: Training the individual in population, evaluating fitness.

Step3: If the population corresponding to the best individual fitness function value is set to meet the requirements or the number of iterations is reached, then go to step 6.

Step4: $k=k+1$.

Step5: Apply selection, crossover and mutation operators to generate new population, go to step 3 .

Step6: Select the individual with largest fitness as the best results, and it is the best threshold to segment the image.

Step7: End.

Otsu integrated with AGA flowchart is shown in Fig. 1.

\section{EXPERIMENT RESULTS}

In order to verify the validity of proposed algorithm, Otsu algorithm and AGA-Otsu algorithm are carried out simulation experiments.

Original infrared images are shown in Fig. 2 and Fig. 3. Image segmentation has made by Otsu based on adaptive genetic algorithm and Otsu respectively. Adaptive genetic algorithm parameters settings are as follows: code length is 8 , the number of individuals in population is 20, $P_{c_{1}}=0.9, P_{c 2}=0.6, P_{m_{1}}=0.1$, $P_{m 2}=0.001$, maximum number of iterations is 100 .

The infrared image is then segmentation using the Otsu and AGA-Otsu methods for comparison. Image segmentation results are shown in Fig. 4-Fig. 7.

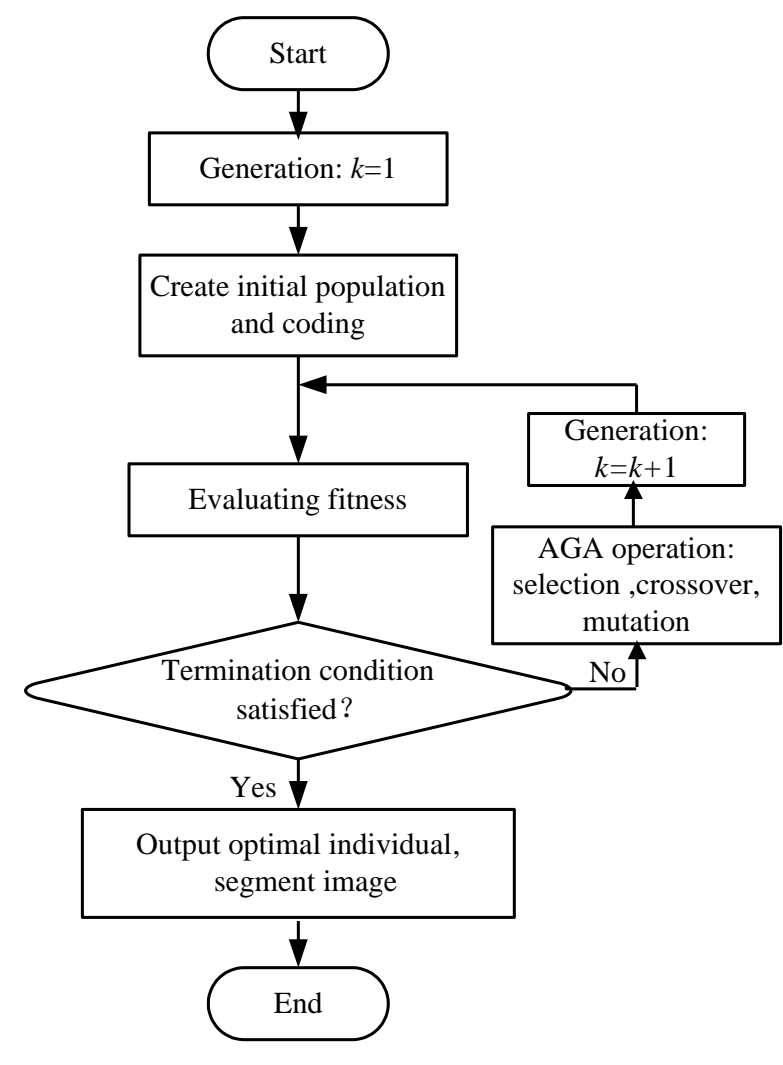

Figure 1. Flowchart of AGA-Otsu

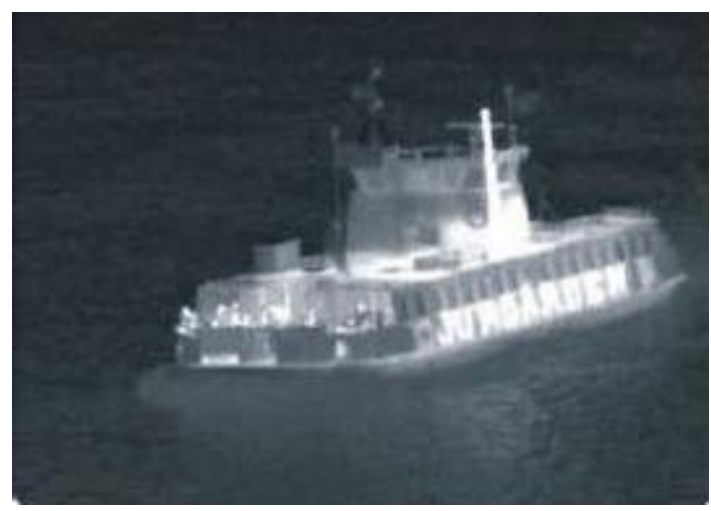

Figure 2. Original infrared image 1 


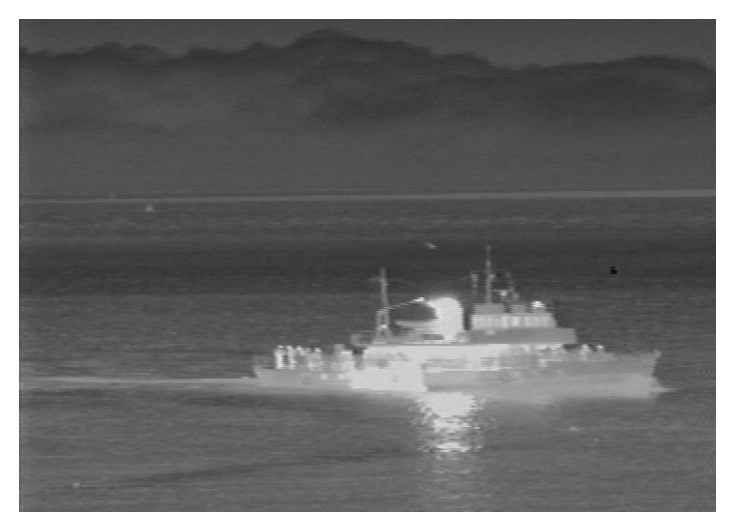

Figure 3. Original infrared image 2

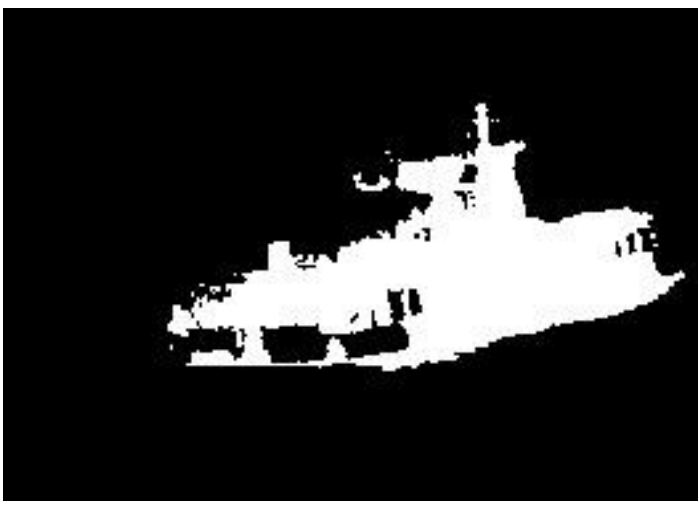

Figure 4. Image segmentation by Otsu for image 1

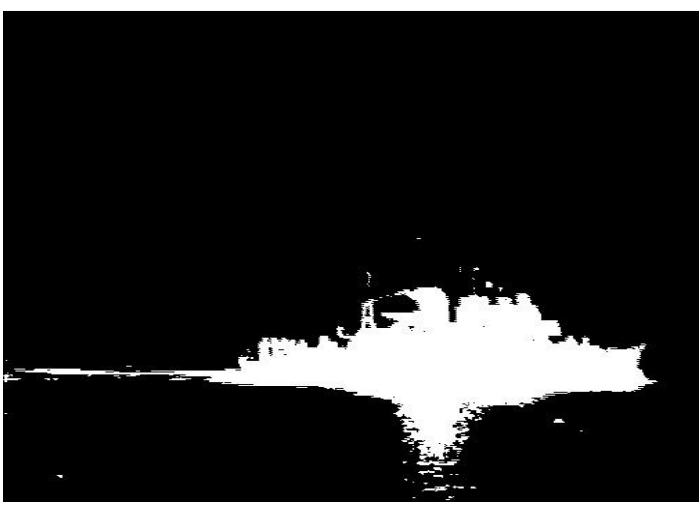

Figure 5. Image segmentation by Otsu for image2

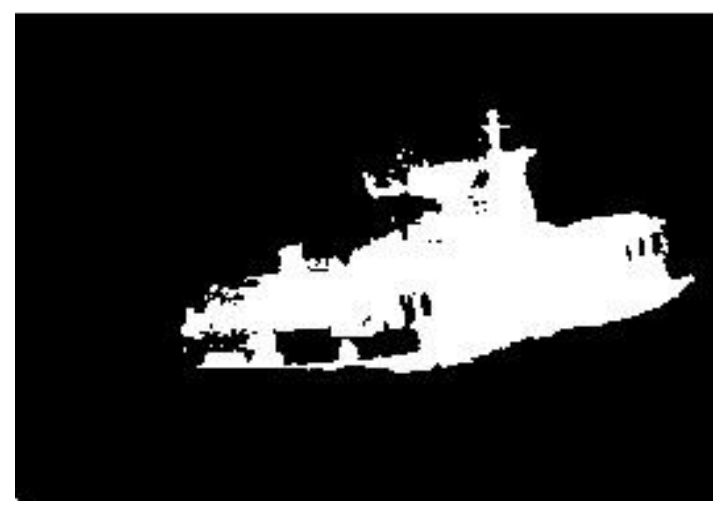

Figure 6. Image segmentation by AGA-Otsu for image1

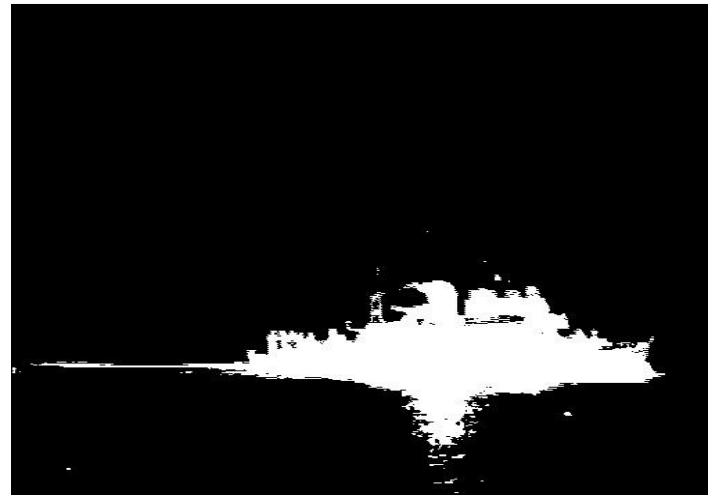

Figure 7. Image segmentation by AGA-Otsu for image2

Fig.4 is Otsu segmentation results of image 1, Fig. 5 is segmentation results of Otsu for Fig. 2. Fig. 6 is AGAOtsu segmentation results of Fig.1 1, Fig. 7 is AGA-Otsu segmentation results of Fig. 2. From Fig. 4 and Fig. 6, Fig. 5 and Fig. 7, it can be seen that the segmentation results of the two methods are similar. Genetic algorithms exist in the process of computing the convergence problems, leading to the results of each operation is different, in order to verify the reliability of the proposed method, by taking the mean of 10 trials way to get this algorithm to determine the optimal threshold. Due to limited space, the paper gives only Fig. 110 times experiment results by AGA-Otsu method, shown in Table 1.

TABLE I. AGA-OTSU METHOD RESULT OF THRESHOLD AND CALCULATION TIME FOR IMAGE 1

\begin{tabular}{|c|c|c|c|}
\hline Methods & Times & Threshold & Time (ms) \\
\hline \multirow{7}{*}{ AGA-Otsu } & 1 & 114 & 123.323 \\
\cline { 2 - 4 } & 2 & 102 & 116.160 \\
\cline { 2 - 4 } & 3 & 129 & 125.115 \\
\cline { 2 - 4 } & 4 & 124 & 136.995 \\
\cline { 2 - 4 } & 5 & 122 & 121.136 \\
\cline { 2 - 4 } & 6 & 112 & 124.965 \\
\cline { 2 - 4 } & 7 & 108 & 124.235 \\
\cline { 2 - 4 } & 8 & 117 & 131.161 \\
\cline { 2 - 4 } & 9 & 120 & 112.104 \\
\cline { 2 - 4 } & 10 & 104 & 133.182 \\
\hline
\end{tabular}

Table 2 compares the performance of the Otsu algorithm and AGA-Otsu algorithm.

TABLE II. Two Methods Performance AND CALCUlation TIME COMPARISON

\begin{tabular}{|c|c|c|c|}
\hline & Methods & Threshold & Time (ms) \\
\hline \multirow{2}{*}{ Image 1 } & Otsu & 118 & 407.023 \\
\cline { 2 - 4 } & AGA-Otsu & 115 & 124.837 \\
\hline \multirow{2}{*}{ Image 2 } & Otsu & 123 & 439.599 \\
\cline { 2 - 4 } & AGA-Otsu & 130 & 121.279 \\
\hline
\end{tabular}

Table 2 shows comparison of the proposed algorithm with the Otsu algorithm computation time and 
segmentation threshold. From Table 1, it can be seen that AGA-Otsu algorithm calculate the segmentation threshold and traditional Otsu algorithm calculate threshold are similar, but in the computation time AGA-Otsu algorithm is significantly better than the traditional Otsu algorithm, the traditional Otsu algorithm computation time is about four times of the AGA-Otsu algorithm.

\section{CONCLUSIONS}

The Otsu algorithm has the attributes of good segmentation effect and easy implementation. But its application is limited by its shortcomings like large amount of computation and long execution time. To solve this problem, a new approach Otsu combined with adaptive genetic algorithm is proposed. Adaptive genetic algorithm is a type of GA of which probabilities of crossover and mutation are adaptively adjusted according to the fitness values of individuals, the goals with adaptive probabilities of crossover and mutation are to maintain the genetic diversity in the population and prevent the genetic algorithms to converge prematurely to local minima. Experimental results show that the proposed algorithm can obtain segmentation results similar to the original Otsu algorithm, better than Otsu but with higher efficiency and less execution time.

\section{ACKNOWLEDGMENT}

This work is supported by National Natural Science Foundation (NNSF) of China under Grant 51409062, 51409064, the Fundamental Research Funds for the Central Universities (HEUCF041501).

\section{REFERENCES}

[1] M. Portes de Albuquerque, I. A. Esquef, A. R. Gesualdi Mello, "Image thresholding using Tsallis entropy," Pattern Recognition Letters, vol. 25, 2004, pp. 1059-1106.

[2] N. R. Pal and S. K. Pal, "A review on image segmentation techniques," Pattern Recognition, vol. 26, 1993, pp. 1277-1294.
[3] N. Otsu, "A threshold selection method from gray-level histogram,” IEEE Trans Systems Man Cybenet, 1979, pp. 62-66.

[4] J. Kittler, J. Illingworth, "Minimum Error Thresholding," Pattern Recognition, vol. 19, 1986, pp. 41-47.

[5] J. Liu, J. H. Zheng, Q. H. Tang, "Minimum error thresholding segmentation algorithm based on 3d grayscale histogram, Mathematical Problems in Engineering, 2014, pp. 1-13.

[6] J. W. Long, X. J. Hen, H. P. Chen, "Adaptive minimum error thresholding algorithm, ”Acta Automatica Sinica, vol. 38, 2012, pp. 1134-1144.

[7] C. Li, Y. Li, X. Wu, "Novel Fuzzy C-Means Segmentation Algorithm for Image with the Spatial Neighborhoods,' International Conference onRemote Sensing, Environment and Transportation Engineering (RSETE), 2012, pp. 1-4.

[8] W. P. Ma, Y. Y. Huang, H. Li, "Image Segmentation Based on Rough Set and Differential Immune Fuzzy Clustering Algorithm," Journal of Software, vol. 25, 2014, pp. 2675-2689.

[9] C. H. Ding, L. Jiang, F. H. Du, "Segmentation of color image contour using fuzzy cluster analysis," International Conference on Information Sciences and Interaction Sciences, 2010, pp. 431-435.

[10] Zhang Gui-Mei, Chen Shao-Ping, Liao Jia-Ni, "Otsu image segmentation algorithm based on morphology and wavelet transformation," International Conference on Computer Research and Development, vol. 1, 2011, pp. 279-283.

[11] Chang, Tsun-Wei, "A GA-based fuzzy recommender system for region-based image retrieval," International Journal of Fuzzy Systems, vol. 16, 2014, pp. 290-302.

[12] Fan SuLing, "GA optimization model for repetitive projects with soft logic," Automation in Construction, vol. 21, 2012, pp. 253-261.

[13] D Adler, "Genetic algorithms and simulated anneling,"A Marriage Proposal In IEEE Confernce on Neural Networks, San Francisco, California, 1993, New York: IEEE Neural Networks Coucil, 1104 1109.

[14] J Andre, P Siarry, T Dognon, “An improvement of the standard genetic algorithm fighting premature convergence in continuous optimization," Advances in Engineering Software, vol. 1, 2011, pp. 49-60.

[15] M. SRINIVAS, L M. PATNAIK, "Adaptive probabilities of crossover and mutation in genetic algorithm," IEEE Transactions on SMC, vol. 24, 1994, pp. 656-667. 
